\title{
Evolução dos Aparelhos Fotopolimerizadores - Revisão de Literatura
}

\author{
Evolution of Light Curing Devices - Literature Review
}

Evolución de los dispositivos de curado por luz - revisión de la literatura

\section{Clara Furtado Granadeiro ${ }^{1 *}$, Luiz Felipe Gilson de Oliveira Rangel ${ }^{2}$, Luís Felipe Coutrin de Toledo ${ }^{3}$, Rodrigo Simões Oliveira ${ }^{4}$}

Como citar esse artigo. Granadeiro, C. F; Rangel, L. F. G. O; de Toledo, L. F. C; Oliveira, R. S. Evolução dos Aparelhos Fotopolimerizadores - Revisão de Literatura. Revista Pró-UniverSUS. 202 Jul./Dez.; 12 (2): 60-64.

\section{Resumo}

A criação da resina composta autopolimerizavel e fotopolimerizavel foi um marco muito importante dentro da odontologia restauradora, possibilitando ao paciente uma restauração mais estética e semelhante ao dente. Com o avanço do século, resinas fotopolimerizáveis estão sendo cada vez mais utilizadas nos consultórios odontológicos, sendo assim aparelhos fotopolimerizadores com luz azul visível viraram uma peça indispensável em um tratamento restaurador. No mercado atualmente possuem unidades de fotopolimerização formadas por diversos princípios físicos, como por exemplo: quartzo-tunsgêniohalogênio (QTH), laser, luzes de arco de plasma e diodos emissores de luz (LED). O objetivo desse estudo é apresentar a evolução dos aparelhos fotopolimerizáveis e como é extremamente importante que o cirurgião dentista conheça mais sobre o aparelho utilizado no seu dia a dia, mostrando suas vantagens e desvantagens e como isso pode afetar o resultado do tratamento restaurador. Para esta revisão de literatura, foi realizado uma busca nas seguintes plataformas, Google Acadêmico, Pub med, Sielo e Elsevier, onde foram selecionados 14 artigos e estudos científicos, dos anos 2005 a 2020, utilizando os descritores: luz LED, avanços da fotopolimerização, polywave, luz halógena, geração dos fotopolimerizadores. Mostrando que atualmente a fotopolimerização feita através de aparelhos com luz LED, tem tomado mais espaço em ambientes odontológicos, podendo ter melhores resultados em uma restauração com resina composta.

Palavras-chave: Polimerização; Cura Luminosa de Adesivos Dentários; Estética Dentária.

\begin{abstract}
The creation of the self-curing and light-curing composite resin was a major milestone in restorative dentistry, allowing the patient to have a more aesthetic and tooth-like restoration. With the advance of the century, photopolymerizable resins are being used more and more in dental offices, so photopolymerizing devices with visible blue light have become an indispensable part of restorative treatment. Currently, on the market, they have photopolymerization units formed by several physical principles, including quartz-tungsten-halogen (QTH), laser, plasma arc lights and light-emitting diodes (LED). The objective of this study is to present the evolution of light-curing devices and how extremely important it is that the dental surgeon knows more about the device used in their day-to-day lives, showing its advantages and disadvantages and how it affects the final result of your restorative treatment. To carry out this literature review, research was carried out on the following platforms, Google Academico, Pub med, Sielo and Elsevier, where 14 scientific articles and studies were selected, from the years 2005 to 2020 , using the descriptors, advances in photopolymerization, polywave, halogen light, generation of light-curing and dentistry and photopolymerization. Showing that currently photopolymerization through LED light devices has taken more space in dental environments and may have better results in restoration with composite resin.
\end{abstract}

Keywords: Polymerization; Light-Curing of Dental Adhesives; Esthetics; Dental.

${ }^{1}$ Discente do Curso de Graduação em Odontologia da Universidade de Vassouras, Vassouras, RJ, Brasil. furtado_clara21@yahoo.com.br. ORCID: https://orcid.org/0000-0002-58389018

${ }^{2}$ Docente do Departamento de Endodontia do Curso de Graduação em Odontologia da Universidade de Vassouras, Vassouras, RJ, Brasil. dr.feliperangel@uol.com.br. ORCID: https:// orcid.org/0000-0002-7376-2829.

${ }^{3}$ Docente do Departamento de Endodontia do Curso de Graduação em Odontologia da Universidade de Vassouras, Vassouras, RJ, Brasil. odontoledo@hotmail.com. ORCID: https:// orcid.org/0000-0003-2131-5549.

${ }^{4}$ Docente do Departamento de Dentística do Curso de Graduação em Odontologia da Universidade de Vassouras, Vassouras, RJ, Brasil. rodsimol@gmail.com. ORCID: https://orcid. org/0000-0003-2347-8608.

* Email de correspondencia: furtado_clara21@yahoo.com.br

Recebido em: 16/09/21. Aceito em: 17/11/21 


\section{Resumen}

La creación de la resina compuesta autopolimerizable y fotopolimerizable fue un hito importante en la odontología restauradora, permitiendo al paciente tener una restauración más estética y parecida a un diente. Con el avance del siglo, las resinas fotopolimerizables se están utilizando cada vez más en los consultorios dentales, por lo que los dispositivos fotopolimerizantes con luz azul visible se han convertido en una parte indispensable en un tratamiento restaurador. Actualmente en el mercado cuentan con unidades de fotopolimerización formadas por varios principios físicos, entre ellos cuarzo-tungsteno-halógeno (QTH), láser, luces de arco de plasma y diodos emisores de luz (LED). El objetivo de este estudio es presentar la evolución de los dispositivos de fotopolimerización y lo sumamente importante que es que el cirujano dental sepa más sobre el dispositivo que utilizan en su día a día, mostrando sus ventajas y desventajas y cómo afecta el resultado final de su tratamiento restaurador. Para realizar esta revisión de la literatura se realizó una búsqueda en las siguientes plataformas, Google Academico, Pub med, Sielo y Elsevier, donde se seleccionaron 14 artículos y estudios científicos, de los años 2005 a 2020, utilizando los descriptores, avances en fotopolimerización, polywave, luz halógena, generación de fotopolimerizadores y odontología y fotopolimerización. Está demostrado que actualmente la fotopolimerización mediante aparatología con luz LED há ocupado más espacio en entornos dentales y puede tener mejores resultados en una restauración con resina compuesta.

Palabras clave: Polimerizacion; Curación por Luz de Adhesivos Dentales; Estética Dental.

\section{Introdução}

Nas décadas passadas, quando se falava de restauração, os materiais mais utilizados eram cimentos de silicato e as resinas, constituídas por matriz orgânica, partículas de carga inorgânica e agente de união ativadora e inibidora de polimerização, sendo recomendado para dentes anteriores ${ }^{1}$. Com a demanda do mercado as primeiras resinas compostas foram lançadas agindo por polimerização química onde se tinha duas pastas, uma pasta universal ou base e outra pasta catalisadora, onde misturando as duas obtinha-se resina autopolimerizável, porém com as dificuldades quanto a manipulação, os resultados alcançados não foram satisfatórios. Surgindo assim a necessidade de criar uma resina composta fotopolimerizável, onde o próprio profissional realiza a polimerização do material restaurador ${ }^{1}$.

A polimerização é o endurecimento das resinas compostas, provocada pela conversão dos monômeros em polímeros, reagidos por adição, que se inicia por uma fonte de energia externa, ou seja, a luz nos compósitos fotopolimerizáveis, gerando a necessidade da criação dos fotopolimerizadores, através de luz ultravioleta, luz halógena de quartzo-tungstênio, luz arco de plasma, laser argônio e a luz emitida por diodo mais conhecida como LED ${ }^{1}$.

Desse modo o profissional que possui um fotopolimerizador deve considerar alguns requisitos, por exemplo, comprimento de onda, densidade de potência, presença ou não de filtros, tempo de vida útil, tipo de manutenção, volume de material restaurador a ser fotopolimerizado, fotoiniciador presente no material restaurador, distância do fotopolimerizador em relação ao incremento e cor e grau de translucidez da resina utilizada ${ }^{1}{ }^{2}$.

Dentre as variedades de luzes criadas, a primeira a se destacar foram os aparelhos com lâmpadas halógenas, devido a sua eficiência, com lâmpadas de filamento de tungstênio, filtro para comprimento de onda e fibras ópticas. Logo após os aparelhos a base de laser de argônio e arco de plasma, com o intuito de reduzir o tempo clínico para uma fotopolimerização eficiente. E por fim a luz de LED onde se possui uma combinação de semicondutores no estado sólido, sob forma de cristais de nitrito de gálio, produzindo luz por eletroluminescência ${ }^{1}{ }^{3}$.

Dessa forma, vale enfatizar o quanto é importante que o cirurgião dentista conheça bem o aparelho utilizado em seu consultório, pois uma polimerização adequada resulta em uma restauração bem-sucedida, onde o paciente não vai ter problemas dentais futuros, como por exemplo manchamento e maior probabilidade de infiltração marginal, devido à falta de polimerização nas camadas mais profundas da cavidade ${ }^{2-6}$.

O objetivo desse estudo é apresentar a evolução dos aparelhos fotopolimerizáveis LED e como é extremamente importante que o cirurgião dentista conheça mais sobre o aparelho utilizado no seu dia a dia, mostrando suas vantagens e desvantagens e como isso afeta o resultado final do seu tratamento restaurador.

\section{Metodologia}

Para esta revisão de literatura, foi realizado uma busca nas seguintes plataformas, Google Acadêmico, Pub med, Sielo e Elsevier, onde foram selecionados 14 artigos e estudos científicos, dos anos 2005 a 2020, utilizando os descritores: luz LED, avanços da fotopolimerização, polywave, luz halógena, geração dos fotopolimerizadores.

\section{Resultados e Discussão}

Com o início da utilização do aparelho a base de diodo emissor LED, novas fontes de luz foram sendo colocadas no mercado, com o objetivo de proporcionar uma polimerização mais eficaz das resinas compostas, 
possuindo vantagens sobre a fonte de luz, que até o momento era realizada pela lâmpada halógena ${ }^{7}$.

O êxito de uma restauração com resina composta fotopolimerizável está ligada à qualidade do polímero formado pela fonte de luz, a emissão suficiente de intensidade, o comprimento de onda emitido e o tempo de exposição ${ }^{1-2,8}$. Acarretando na foto sensibilização do principal fotoiniciador, conhecido como Canforquinona ${ }^{1-}$ 2,6-8

Dessa forma a unidade de fotopolimerização dentária (LCU) é essencial para o processo de cura de uma resina composta, porém a falta de conhecimento das reais vantagens e desvantagens passam despercebidos e seus futuros efeitos ignorados ${ }^{2,4,9-10}$.

A polimerização inadequada da resina composta desencadeia problemas ao paciente, devido à falta de polimerização das camadas mais profundas da cavidade, onde ocorre tais fatores como falta de penetração de luz incidente, distância entre a fonte de luz e a resina composta, a condição do aparelho utilizado e o direcionamento da luz $z^{1,4,9,11}$. Por consequência disso o mercado vem a presentando novos aparelhos, atualizados, sendo importante que o profissional avalie as alterações realizadas pelo fabricante ${ }^{2,4-5}$.

Em 1970 foi empregado no mercado o primeiro fotopolimerizador, conhecido como luz halógena de quartzo-tungstênio, sendo a fonte de luz visível mais conhecida no meio odontológico, porém mesmo sendo muitoutilizada, alguns pontos limitavam seu desempenho como, sua vida útil que varia entre 40 a 50 horas, dependendo de quantas vezes o profissional vai utilizar o aparelho ${ }^{1,3,8}$. Possui luz incandescente, devido a isso a energia elétrica era convertida em calor, acarretando pouca durabilidade, provocando a degradação da lâmpada, refletor e filtro, que quando não trocados causam a diminuição da eficácia da polimerização, aumentando o risco de erro na restauração ${ }^{1-4,6,8,10}$. Vale ressaltar que os aparelhos de luz halôgena não alcançam a intensidade de luz mínima registrada pelo fabricante, isso acontece devido à falta de manutenção e a não checagem da intensidade ${ }^{8,10}$. Em relação ao seu espectro de emissão de luz, é grande, entre 380 e 510 nm com seu pico perto de $480 \mathrm{~nm}$. Porém é importante destacar que a luz halôgena é uma tecnologia de baixo custo, possuindo uma boa intensidade de potência e um amplo espectro de luz ${ }^{3,6}$.

Em seguida, fotopolimerizadores a base de laser de argônio e arco de plasma foram lançados no mercado, sua maior proposta era reduzir o tempo clínico de fotopolimerização. Mesmo possuindo uma luz eficiente para fotopolimerizar a resina composta, algumas desvantagens fizeram com que seu uso seja inviável ao profissional, como por exemplo seu alto custo para adquirir o aparelho e sua manutenção, frisando também os possíveis danos ao tecido pulpar, devido ao calor exagerado ${ }^{1}$.
A mais recente tecnologia criada com meio de polimerizar resinas compostas é a luz gerada por LED (luz emitida por diodo), que em relação as outras luzes convencionais destacam-se por ser pequeno e eficiente em energia, com a utilização de bateria de níquelhidreto metálico de alto desempenho (NiMH) ou de ion-lítio $^{12}$. Os LEDs são aparelhos fotônicos fundada em semicondutores In-Ga-N (índio-Gálio-Nitrogênio), onde as partículas de luz conhecidas como fótons executam o papel principal, sendo assim convertem energia elétrica em radiação óptica, onde a luz é emitida através de polarização direta ${ }^{2,12}$.

Para chegada dessa nova unidade de fotopolimerização (LCUs) de LED, seria preciso que dois pré-requisitos fossem efetuados ${ }^{12}$ : Os LEDs azuis precisavam ter o comprimento de onda de emissão apropriado; A energia emitida pelos LEDs azuis deveria ser suficiente para curar materiais dentários, dentro do tempo de 20 a 40 s.

Porém LEDs de primeira geração, não foram bem recomendados, devido a sua emissão de energia, variando entre 75 a $150 \mathrm{~mW} / \mathrm{cm}^{2}$, ou seja, muito pequeno para ser utilizada durante o tempo relativamente considerável clinicamente, onde se era necessário a utilização de vários LEDs para se ter o mesmo efeito que um fotopolimerizador de luz Halógena ${ }^{1,2,7,12}$, sendo necessário melhorias.

Contudo esses aparelhos apresentaram diversas vantagens, como os benefícios extrínsecos, que aumentam a qualidade de fotopolimerização de biomateriais orais. E a largura de onda espectral, sendo 5 a 20nm, gerando como um resultado uma fotopolimerização mais eficaz ${ }^{12}$.

Devido aos benefícios criados com os primeiros LEDs, os LEDs de segunda e terceira geração foram colocados no mercado, apresentando melhorias na sua potência e na profundidade de cura, onde se tem um aparelho eficiente para fotopolimerização, que não causa aquecimento no tecido pulpar e uma maior facilidade em polimerizar incrementos acima de $2 \mathrm{~mm}$ de forma correta sem danos futuros ${ }^{1-4,7-8,10-12}$. Sua eficácia luminosa, também deve ser citada, mostrando o quanto uma fonte de luz produz luz visível de forma correta. Para os LEDs a eficácia luminosa é geralmente de ordem de $60 \mathrm{ImW}_{-1}$, já os LEDs brancos a eficácia luminosa de $150 \mathrm{ImW}_{-1} 12$

Sendo assim os LEDs atuais apresentaram uma ótima eficácia energética onde um aparelho de luz LED opera 25 min em média com a bateria totalmente carregada, sendo então capaz de fotopolimerizar 75 ciclos de 20 segundos $^{12}$, sendo assim o tempo de vida útil do aparelho vai em torno de 100.000 horas, ou até mais dependendo da variação de tempo usada pelo profissional $^{1-4,7-8,10-12}$.

Portanto atualmente no mercado odontológico, possui os LEDs de segunda geração contendo luz azul, 
onde seu pico de radiação chega a 460nm, sendo muito próximo ao espectro de absorção da canforoquinona que é de 468nm, obtendo maior conversão, ou seja, o mais adequado para as principais resinas comerciais ${ }^{12-13}$. E também os LEDs terceira geração como, Polywave que consiste em um LED azul central voltado por quatro LEDs violetas, com comprimentos de onda adequados para fotopolimerizar incrementos que possuem novos fotoiniciadores, como fenilpropodiona (PPD), bisaquil e fosfínico (BAPO) $)^{1,12,14}$, ou seja, são aplicados duas ou mais bandas de comprimento de onda estreito e diferentes que podem partir deste LED, gerando diferentes comprimentos de onda, cada uma com seu máximo distinto ${ }^{12}$. E por fim o Bluephase, que possui duplo comprimento de onda com irradiância máxima de $2200 \mathrm{~mW} / \mathrm{cm}_{-2}{ }^{12,14}$

Sendo assim, o profissional precisa saber qual aparelho mais o ajuda clinicamente, sabendo qual fotopolimerizador garante o melhor resultado no seu trabalho restaurador. Quando não se é considerado essas informações sobre o aparelho, acaba que dispositivos fotopolimerizadores mais potentes, são mais procurados para que assim o tempo de trabalho seja reduzido e consequentemente o paciente tenha mais conforto. Porém devido aos altos valores de irradiância a profundidade de cura pode ser aumentada, ou seja, a energia fornecida ao dente será dobrada, aumentando o calor no tecido pulpar ${ }^{1,12}$

Dessa maneira antes de se usar um fotopolimerizador, clinicamente é necessário o conhecimento sobre o composto a ser curado e se ele possui compatibilidade com o comprimento de onda do aparelho, pois estudos mostram que fotopolimerizadores LED são extremamente eficazes, porém quando não utilizado com fotoiniciador compatível, seus efeitos podem ser negativos, acarretando uma má fotopolimerização $0^{1,12,14}$.

Em comparação com as resinas autopolimerizáveis, resinas compostas possuem maiores vantagens, como por exemplo, menor porosidade e descoloração, fácil manipulação e maior tempo de trabalho. No entanto pode-se afirmar que a dureza no topo dos materiais fotopolimerizáveis, não é garantia de uma polimerização adequada em todo o incremento. Sendo assim, os fotopolimerizadores devem apresentar eficácia na ativação dos fotoiniciadores presentes nas resinas, potencializando as características físicas desses materiais ${ }^{6,10}$.

Em virtude disso, aparelhos a base de LED vem sendo cada vez mais empregados. Isso porque mesmo com a grande variedade, o LED vem se destacando devido a qualidade de luz emitida o que acarreta no maior sucesso clínico em procedimentos restauradores 1 .

Mesmo sendo reprovado inicialmente, as suas inúmeras vantagens como por exemplo: maior tempo de vida útil, a não utilização de filtros, maior durabilidade de bateria, seu aparelho ser pequeno e funcional e além de tudo o fato de emitirem luz azul em uma faixa espectral estreita, sendo compatível a canforoquinona, o principal fotoiniciador das resinas compostas, resultou em novos planejamentos para as novas gerações de fotopolimerizadores a base de LED, podendo também ser utilizados em outros fotoiniciadores presentes no mercado $^{1-2,11-12}$

Consequentemente fatores como, o tipo de fonte de luz, intensidade, comprimento de onda e tempo de exposição, resultaram na polimerização mais profunda das resinas. Isso faz com que haja uma comparação entre as luzes convencionais e as luzes a base de diodo emissor, onde estudos mostram que os LEDs não superaquecem as estruturas dentais e nem o material restaurador, carretando na diminuição da sensibilidade pós-operatória e também causando menor contração de polimerização, sendo possível uma polimerização adequada em incrementos com $2 \mathrm{~mm}$ no tempo ideal de $20 \mathrm{~s}^{3,7}$

Sendo assim o profissional possui uma gama de aparelhos a base de diodo emissor, agregando seu atendimento no consultório. LED de primeira e segunda geração possuindo luz azul, ideal para atingir o pico de absorção da canforoquinona. LED Polywave (terceira geração) desenvolvido devido a criação de novos fotoiniciadores, como por exemplo o PPD (fenilpropodiona), possuindo LED azul e LED violeta e por fim LED Bluephase possuindo comprimento de onda duplo ${ }^{11-13}$.

Portanto a técnica de fotopolimerização realizada com LED se tornou a melhor escolha para a polimerização de compósitos odontológicos. Sendo aparelhos de LED com alta potência e com a melhor eficácia energética apresentada. Tornando-se a melhor escolha para tratamentos restauradores a base de resina fotopolimerizável ${ }^{12}$.

\section{Conclusão}

De acordo com o estudo apresentado, conclui-se que os aparelhos fotopolimerizadores representaram um grande avanço na odontologia restauradora. Onde até o atual momento 3 fontes de luz foram destacadas, sendo elas, Luz Halógena, Laser Argônico de Arco de Plasma, até chegar na luz LED, tornando-se a mais recomendada no mercado. Porém é importante que o profissional conheça o aparelho utilizado no seu consultório, priorizando aquele que melhor se enquadra na resina utilizada pelo mesmo, estando ciente de que o fotopolimerizador tem que ser compatível ao fotoiniciador presente no material restaurador, não o inverso. Isso porque mesmo com as diversas vantagens apresentadas, esse aparelho pode parar de ser vantajoso, 
podendo não polimerizar o incremento corretamente e aumentando o calor no tecido pulpar, causando assim problemas futuros ao paciente

\section{Referências}

1. Cardarelli P.G, Beltrani F.C, Pereira S.K., Cardoso S.A, Hoeppner M.G. Aparelhos fotopolomerizadores: evolução e aplicação clínica - uma revisão de literatura. Odontol. Clín. - Cient. 2011; 10(4): 317-321

2. Lopes L.G, Souza J.B, Andrade F.R, Ferreira P.R, Freitas G.C, Barnabé W, Campos B.B. Profundidade de Polimerização de Resinas Híbrida, Micro e Nanoparticuladas Utilizando Luz Halógena ou Led de Segunda Geração. Robrac. 2006; 15(39): 37-43

3. Accetta D.F, Filho T.R.M, Weig K.M, Fraga R.C. Influência dos fotopolimerizadores (luz halógena x led) na resistência à compressão de resinas compostas. Rev. Fac. Odontol. 2008; 49(3): 17-19

4. Firoozmand L.M, Araujo R.M, Balducci I. Influência de fotopolimerização de luz halôgena $\mathrm{X}$ led azul na dureza de resina composta. Cienc Odontol Bras. 2005; 8 (1): 67-74

5. Price R.B, Ferraccane J.L, Heickel R, Sullivan B. The light-curing unit: An essential piece of dental equipment. FDI World Dental Federation. 2020: 1(1):1-11

6. Reston G, Barbosa E.N, Busato A.S, Jr A.L.K, Carli C.A, Carvalho G.M, Schirmer C, Daniel. Microdureza de resina composta polimerizada com LEDs de diferentes gerações e luz halógena. Stomatos. 2008; 14(27): $17-25$

7. Rastelli A.N.S, Andrade M.F, Kurachi C, Bagnato V.S. Processo de Fotoativação de Resina Composta - Estudo Comparativo entre Nova Geração de Led's e a Luz Halógena. RGO. 2006; 54 (2): 139-143

8. Bosquiroli V, Franco E.B, Pereira J.C, Navarro M.F.L, Mondelli R.F.L. Fotopolimerização de Resina Composta com Luz Halógena e LED. RGO. 2006; 54(2): 129-133

9. Preço R.B, Ferreacane J.L, Shortall A.C. Light-Curing Units: A review of What We Need to Know. Journal of Dental Research. 2015; 94(9): 1179-1186

10. Soares C.C.P, Peçanha M.M, Batitucci R.G, Neto R.G, Batitucci E, Batitucci M.H.G. Eficácia da polimerização de uma resina composta fotopolimerizada por aparelhos de luz halógena e LED da clínica integrada do curso de odontologia da UFES. EFES Rev. Odontol. 2005; 7(3): 58-65

11. Cadenaro M, Maravic T, Comba A, Mazzoni A, Fanfoni L, Hilton T, Ferracane J, Breschi L. The role of polymerization in adhesive dentistry. Dental Materials. 2019; 35(1): 1-22

12. Jandt K.D, Mills R.W. A brief history of LED photopolymerization. Dental Materials. 2013; 2(9): 605-617

13. Chen Y, MDS, Yao C, PhD, Huang C, PhD, Wang Y, PhD. The effect of monowave and polywave light-polymerization units on the adhesion of resin cements to zirconia. JPD. 2019; 1(1): 1-7

14. Arikawa H, Takahashi H, Kanie T, Ban S. Effect of various visible light photoinitiators on the polymerization and color of light-activated resins. Dental Materials Journal. 2009; 28 (4): 454-460. 